\title{
Vibration-based Fault Diagnostic of a Spur Gearbox
}

\author{
Dennis Hartono ${ }^{1}$, Dunant Halim ${ }^{1}$, Gethin Wyn Roberts ${ }^{2}$ and Qianyu Liu ${ }^{1}$ \\ ${ }^{1}$ Department of Mechanical, Materials and Manufacturing Engineering The University of Nottingham Ningbo China, China 315100 \\ ${ }^{2}$ Department of Civil Engineering, The University of Nottingham Ningbo China, China 315100
}

\begin{abstract}
This paper presents comparative studies of Fast Fourier Transform (FFT), Short Time Fourier Transform (STFT) and Continuous Wavelet Transform (CWT) as several advanced time-frequency analysis methods for diagnosing an early stage of spur gear tooth failure. An incipient fault of a chipped tooth was investigated in this work using vibration measurements from a spur gearbox test rig. Time Synchronous Averaging was implemented for the analysis to enhance the clarity of fault feature from the gear of interest. Based on the experimental results and analysis, it was shown that FFT method could identify the location of the faulty gear with sufficient accuracy. On the other hand, Short Time Fourier Transform method could not provide the angular location information of the faulty gear. It was found that the Continuous Wavelet Transform method offered the best representation of angle-frequency representation. It was not only able to distinguish the difference between the normal and faulty gearboxes from the joint angle-frequency results but could also provide an accurate angular location of the faulty gear tooth in the gearbox.
\end{abstract}

\section{Introduction}

Vibration signals generated from a gearbox contain abundant information about the operating condition and health of the gearbox. However, the underlying vibration signal is generally very complex and specialized signal treatments using certain signal processing methods will be needed. It is well known that impulse-like vibration signal is generated when a fault occurs in a gearbox. Impulse is a short duration event that excites vibration over a wide range of frequencies. It can be considered as a transient and a non-stationary signal. However, most signal processing methods used to analyze vibration signal are holding on the assumption that the signal is stationary, For example, Cepstral analysis, FFT, and the amplitude or frequency demodulation have been applied for gearbox fault diagnosis $[1,2]$. Thus, more sophisticated signal processing methods such as beyond the classical Fourier Transform approach are needed to achieve reliable and accurate gearbox diagnosis. A relatively small number of advanced time-frequency methods have been used to analyse non-stationary signals such as The Short Time Fourier Transform, Continuous Wavelet Transform and Wigner Ville Distribution, although they have not been implemented as rigorously as stationary signal processing methods in the condition monitoring industry [3-5].

In this work, the Time Synchronous Averaging (TSA) technique was initially implemented to extract the fault feature from the vibrations signals that are heavily corrupted by measurement noise. TSA has the capability of presenting the vibration information in terms of angular rotation of gear, enabling the comparison between the healthy and faulty gears. FFT was then implemented to the TSA signal to extract the sidebands around gearmesh frequencies that indicate the fault location [6]. However, FFT only could provide the frequency content of the TSA signals and the angular information of the faulty gear is lost. To overcome this drawback, the use of STFT and CWT were proposed in this work to further analyze the TSA signal for accurate diagnostic purposes. This analysis can reveal both the frequency content of the TSA signals and also indicate how the frequency content evolves with respect to the angular location of the gear. Such information can also be used to determine the gear fault location in the angular domain.

This paper is organized as follows. Section 2 provides a brief description about the background theory of TSA, STFT and CWT methods, while Section 3 presents the experimental set-up using a spur gearbox test rig developed at the University of Nottingham Ningbo china. Section 4 discusses the experimental results based on the vibration measurement from the gearbox, and finally Section 5 offers the main conclusion of this work.

\section{Theoretical background}

\subsection{Time synchronous averaging}

Time Synchronous Averaging (TSA) is commonly used to extract the vibration of a single gear from the overall vibration of the gearbox system, which is quite useful for 
diagnostic purposes. In this situation, the gears are supposed to be operated at constant load and speed, and all the teeth of the gears can be considered to be identical and equally spaced. The meshing vibration $\mathrm{x}(t)$ is thus determined by the signal average over one of the gears with $T$ teeth and rotates at $f s \mathrm{~Hz}$ as formulated as:

$$
\mathrm{x}(t)=\sum_{m=0}^{M} X_{m} \cos \left(2 \pi m T f_{s} t+\emptyset_{m}\right)
$$

where $X_{m}$ is the amplitude of the meshing vibration, $f_{\mathrm{s}}$ is the rotational frequency of the gear, $\emptyset_{m}$ is the inital phase. If one of the teeth has a local defect such as a chipped tooth, it will cause amplitude and phase modulations. The resulting modulated gear meshing vibration is formulated as [7]:

$$
\mathrm{g}(t)=\sum_{m=0}^{M} X_{m}\left(1+a_{m}(t)\right) \cos \left(2 \pi m f_{s} t+\emptyset_{m}+b_{m}(t)\right)
$$

where the amplitude modulation function and the frequency modulation function, $a_{m}(t)$ and $b_{m}(t)$, can be formulated as:

$$
\begin{aligned}
& a_{m}(t)=\sum_{n=0}^{N} A_{m n} \cos \left(2 \pi n f_{s} t+\alpha_{m n}\right) \\
& b_{m}(t)=\sum_{n=0}^{N} B_{m n} \cos \left(2 \pi n f_{s} t+\beta_{m n}\right)
\end{aligned}
$$

Here, $a_{m}$ and $b_{m}$ are the amplitude and phase modulation caused by the changes in vibration signals due to the defect. $\alpha_{m n}$ and $\beta_{m n}$ are the phase angle of amplitude and phase modulation functions respectively. $A_{m n}$ and $B_{m n}$ are the amplitude of each harmonic in the amplitude and phase modulation function respectively. The index $m$ indicates that the modulation function may differ for each harmonics $m$. The index $n$ represents the harmonic index of the amplitude and phase modulation functions with respect to the gear shaft rotation frequency $\left(f_{s}\right)$.

\subsection{Short time fourier transform}

The basic idea of STFT is slicing the signal into different segments (with possible overlaps). Assuming that within each slice/segment the underlying signal will behave as a stationary signal, then each slice can be subjected to Fourier Transform analysis. It can be formulated as [8]:

$$
\mathrm{X}(t, f)=\int_{-\infty}^{\infty} \mathrm{x}(\tau) \mathrm{w}(t-\tau) \mathrm{e}^{-j \pi f \tau} d \tau
$$

where $\mathrm{w}(t-\tau)$ is the window function used and $\tau$ is the variable that slides the window across the underlying waveform.

\subsection{Continuous wavelet transform}

Continuous Wavelet Transform is another type of linear transformation of a signal based on decomposition of the signal in terms of a basis of elementary functions. It is formulated as $[9,10]$ :

$$
W_{g}(a ; b)=\frac{1}{\sqrt{a}} \int_{-\infty}^{\infty} \mathrm{x}(t) \mathrm{g}^{*}\left(\frac{t-b}{a}\right) d t
$$

where $\mathrm{g}(t)$ is the analysing wavelet that is translated and dilated by parameter $b$ and $a$ respectively

$$
g_{a, b}(t)=\frac{1}{\sqrt{a}} h^{*}\left(\frac{t-b}{a}\right)
$$

There are a number of different real and complex valued functions for mother wavelet. Morlet wavelet has been proven to be the most suitable for gearbox fault diagnosis [11]. It can be formulated as [12]:

$$
\psi(x)=\frac{1}{\sqrt{\pi F b}} e^{2 i \pi F_{c} x} \mathrm{e}^{\frac{-x^{2}}{F b}}
$$

where $F_{b}$ is the bandwidth parameter and $F_{c}$ is the centre of the morlet wavelet.

\section{Gearbox experimental setup}

A spur gearbox test rig was used for this work as presented in Fig. 1. During the experiment, a 1 HP DC motor was used as the driver of the system. The rotational speed of the input was set at 500 RPM, corresponding to the frequency of $8.37 \mathrm{~Hz}$. Two torque and speed sensors located at the input and output of the gearbox, were incorporated to ensure the constant operational speed during the experiment. There was no load applied to the gearbox for this experiment although additional load could be provided using a magnetic brake attached to the test rig. The whole experiment was conducted on the Nexus Optical Table to isolate vibration from external environment.

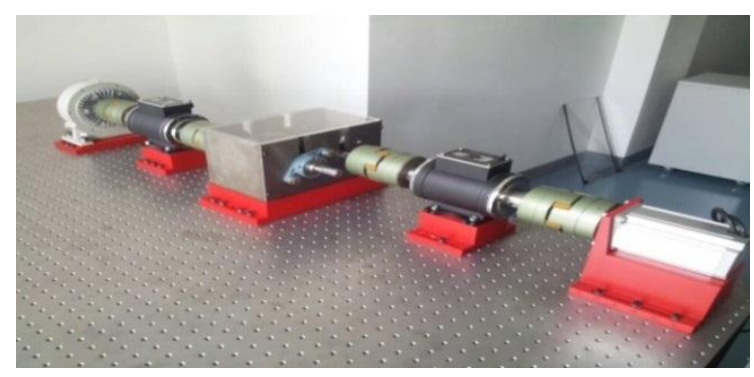

Figure 1. The gearbox test rig used for experiments.

The experimental data were obtained from a spur gearbox comprising a pinion gear of 18 teeth in mesh with another gear of 27 teeth, driven by the DC motor. The faulty tooth considered in this work was a chipped tooth as shown in Fig. 2, where it can be seen that only a slight part of the gear has been taken out. This was done in purpose to test whether the proposed signal processing algorithm was sensitive enough to detect such a small incipient failure in gearbox.

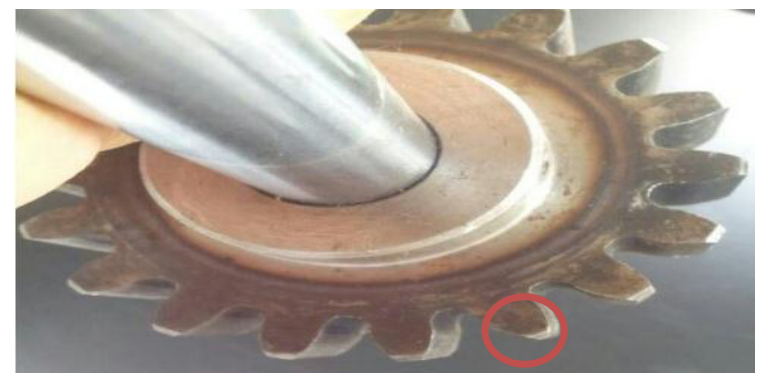

Figure 2. The gear with faulty tooth. 
In this work, three accelerometers were placed on a bearing housing as depicted in Fig. 3. However, only vibration measurement in the radial direction was analyzed further in this work. NI CompactRio was used for data acquisition with 9234 module and $5.120 \mathrm{kHz}$ sampling rate was used to obtain 200000 data samples from vibration measurement. In addition, a tachometer sensor was also used to process the raw vibration data using Time Synchronous Averaging.

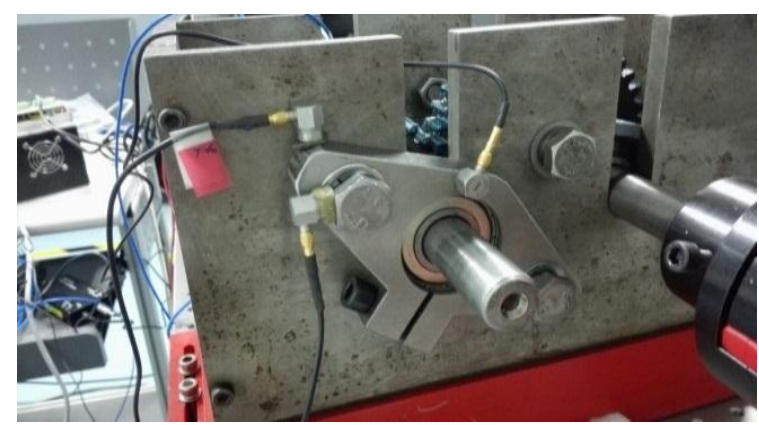

Figure 3. Accelerometers location on the bearing housing.

\section{Results and discussion}

Vibration signal, in terms of radial acceleration, from a healthy and faulty gearboxes are presented in Figs. 4 and 5. As observed from the figures, it is difficult to differentiate vibration signals obtained from the healthy or faulty gearboxes based solely on evaluating the raw vibration signals. The only thing that can be observed is that there is a slight increase of the vibration amplitude of the faulty gearbox compared to that of the healthy gearbox. This can be explained by the fact that at the early stage of the gear fault, relatively small impulse responses are generated. This makes the amplitude of vibration signal from the faulty gearbox almost indistinguishable compared to that of the healthy gearbox.

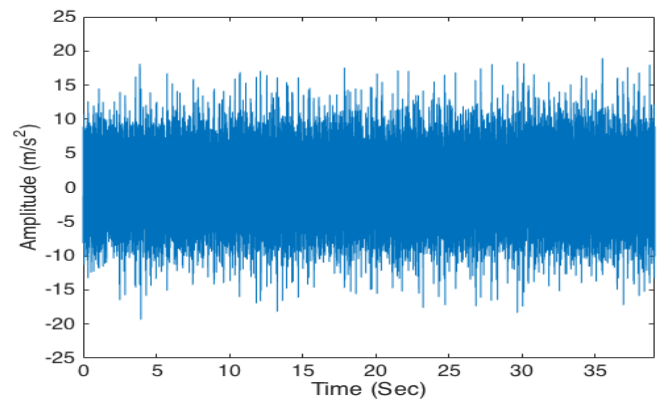

Figure 4. Vibration signal from a healthy gearbox

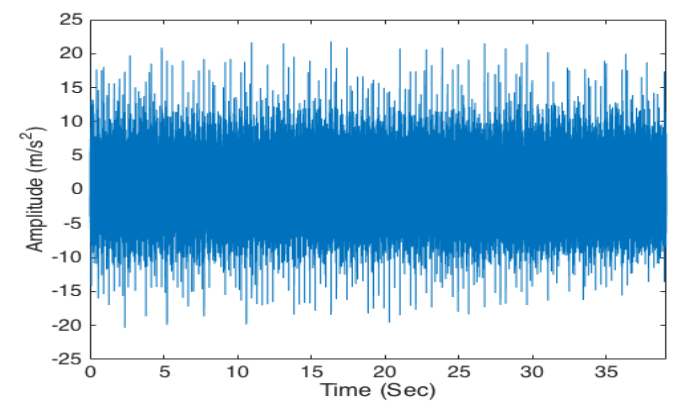

Figure 5. Vibration signal from a faulty gearbox
From the results, a large number of spikes were also observed in the vibration signals from both healthy and faulty gearboxes. The spikes that came from the faulty gear could be used to identify which gear contained the fault by identifying the periodicity patterns of signal [7]. However, it was found that it was not possible to detect the periodicity from the time domain plot itself. Not only that the background noise that made it difficult to observe the signal periodicity, but also the the nature of the gearbox vibration signal itself that was rather complex due to vibration contributed by various components, such as gearbox housing. Therefore, an advanced preprocessing tool was required to filter out the signal from the asynchronous signal components and background noise. In this work, TSA was proposed as a preprocessing tool to eliminate the asynchronous signal component and enhance the signal to noise ratio of the vibration signals.

TSA vibration signal from a healthy gearbox is presented in Fig. 6. From the results, it is clear how the background noise in vibration signal has been diminished significantly, compared with the raw vibration signals shown in Fig. 4. It was shown that TSA was not only able to reduce the background noise that corrupted the vibration measurement, but it could also eliminate asynchronous signal components captured by the accelerometer, so that it could identify the fault feature more accurately. It was also observed that the amplitude of the TSA signal showed a regular pattern that could be analysed further.

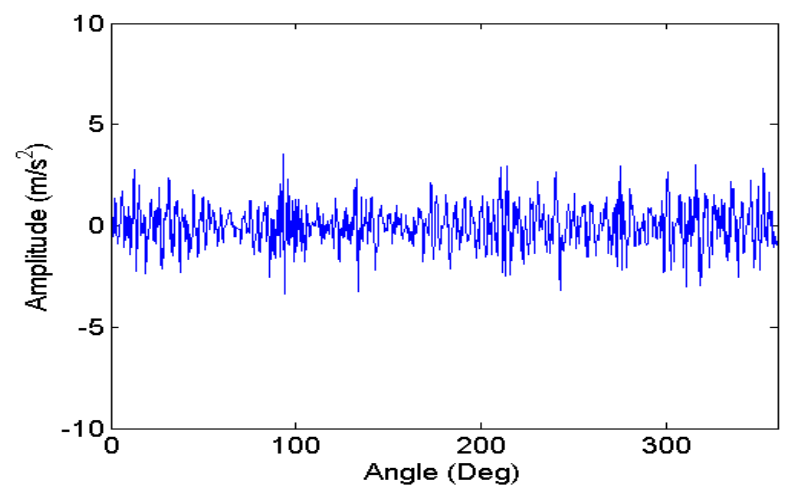

Figure 6. TSA vibration signal from a healthy gearbox

Moreover, based on the performed analysis, it was found that the TSA processing was able to extract the weak impulse signal generated from a faulty gearbox that was not possible to observe from the raw vibration signal, as shown Fig. 7. The results indicate that there is a relatively large spike observed in the signal from a faulty gearbox, which was located at a particular gear angle of around $270^{\circ}$ as observed in Fig. 7. This spike was associated with the impulse response generated from the faulty gear located at angle of $270^{\circ}$.

The results demonstrated that TSA was able to identify the angular location of the faulty gear tooth with sufficient accuracy. This was done by determining the angular location of the impulse response generated by the faulty gear. However, the method could not be used to identify which particular gear had the faulty tooth In this 
case, spectral analysis method could be used. FFT of TSA signal from a healthy gearbox is presented in Fig. 6 . As expected from a normal gearbox, several peaks were observed at the gearmesh frequency and its harmonics with low amplitude sidebands around them. These sidebands were the fault indicator that would be compared with the FFT plot from the faulty gearbox.

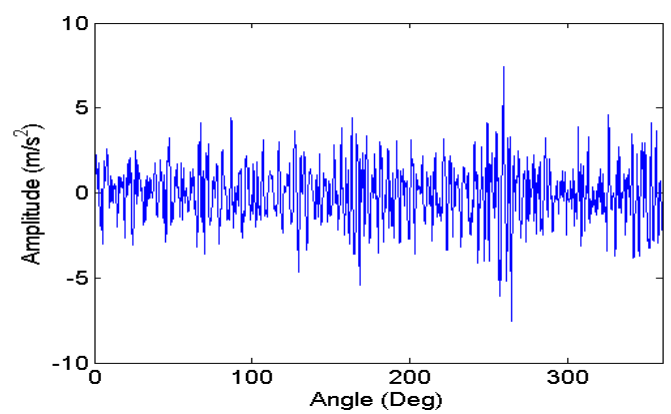

Figure 7. TSA vibration signal from a faulty gearbox

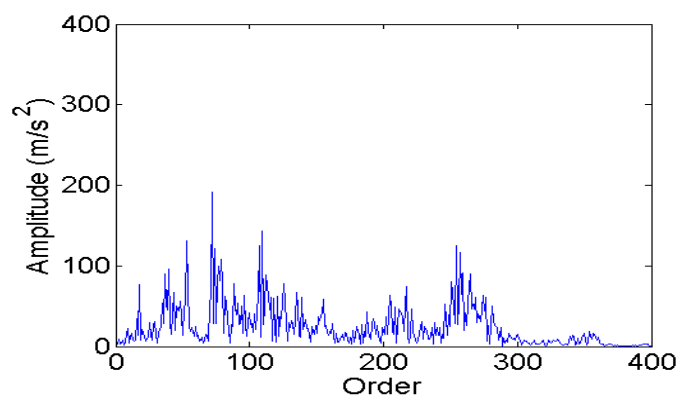

Figure 8. FFT vibration signal from healthy gearbox

The FFT of the faulty gearbox is presented in Fig. 8. Compared to the FFT of the healthy gearbox, an increase of amplitude in several gearmesh frequencies and sidebands around them could be observed. Sidebands are considered as a useful indicator to provide information about the location of the faulty tooth from its spacing from the gearmesh frequency [6]. However, FFT is not suitable to analyze the transient signal such as the impulse vibration signal generated from a faulty gearbox, due to its stationary assumption of the underlying signal. From the experimental results, it was quite difficult to see the sidebands spacing because they were corrupted by the background noise. In addition, there is an inherent drawback of the FFT due to the spectral leakage that will smear the spectral peaks. Furthermore FFT is only able to present the information in frequency domain and cannot provide the time domain useful for fault diagnostic purposes.

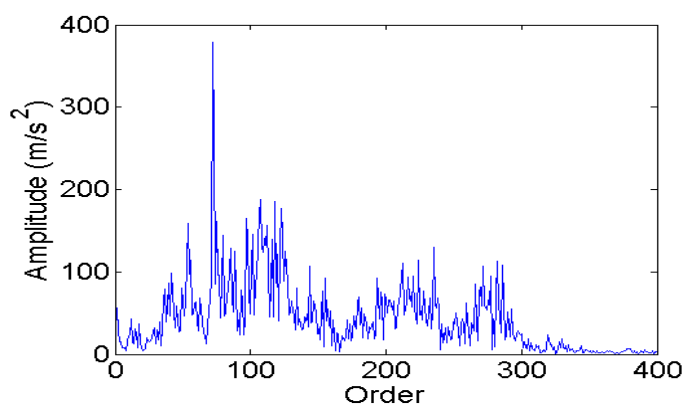

Figure 9. FFT vibration signal from a faulty gearbox
Thus, another time-frequency signal processing method was investigated in this work. STFT of TSA signal from healthy and faulty gearboxes are presented in Figs. 6 and 7. From the results, it was clearly observed the contrast of intensity between TSA signals from the healthy and faulty gearboxes. However, the results were not specific enough to provide the angular location of the faulty tooth accurately. As observed in Fig. 10, STFT provided a fault indication by indicating strong impulse signals around $160^{\circ}$ and $340^{\circ}$, whereas the actual faulty tooth only occurred at approximately $270^{\circ}$. This result can be explained by the fact that STFT requires an appropriate selection of parameter for its window function. Once it is chosen, it will be used in the entire analysis since STFT has a constant time-frequency resolution in the entire time-frequency plot. Therefore it is not suitable for analyzing gearbox vibration signals that contain multi-scale components [13].

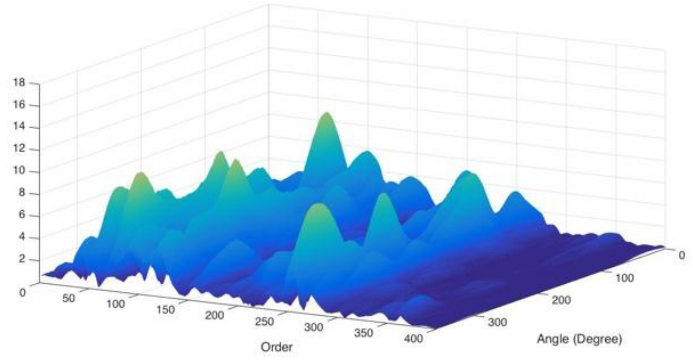

Figure 10. STFT of TSA vibration signal from a healthy gearbox

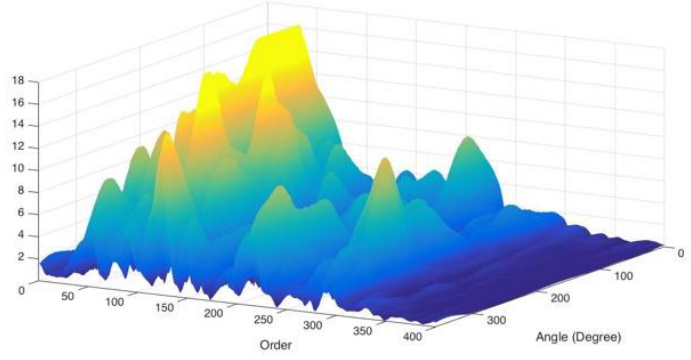

Figure 11. STFT of TSA vibration signal from a faulty gearbox

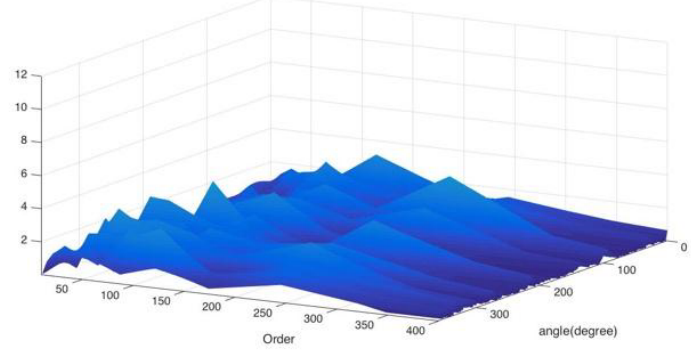

Figure 12. CWT of TSA vibration signal from a healthy gearbox

From the drawback of STFT method, an alternative CWT method was later implemented in this work. The plot of the CWT from healthy and faulty TSA signals from the gearbox are presented in Figs. 12 and 13. Based on the results, it was demonstrated that CWT was not only able to distinguish the TSA vibration signal from 
healthy and faulty gearboxes, but also provided an accurate angular location where the fault occured compared to STFT. CWT was able to pinpoint the strong frequency component occurred around $270^{\circ}$, as also correctly identified from the TSA results in Fig. 7.

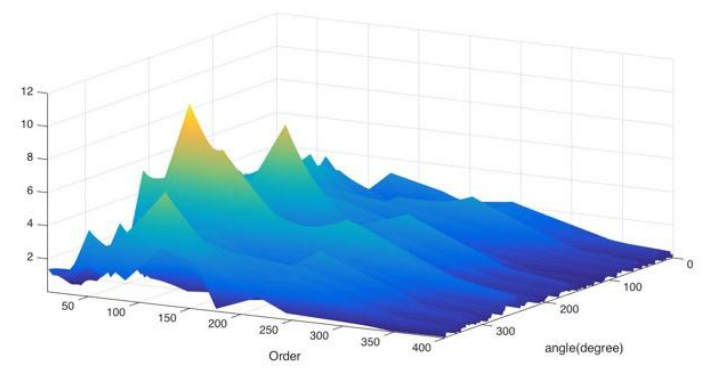

Figure 13. CWT of TSA vibration signal from a faulty gearbox

\section{Conclusions}

Vibration-based fault diagnostic of a gearbox has been presented in this work using experimental measurements from a spur gearbox test rig. The work compared FFT, STFT and CWT as several advanced signal processing methods for analyzing the non-stationary signal associated with an incipient gear fault in the form of a chipped tooth. TSA was initially implemented to enhance the meshing vibration of the gear of interest. It was shown in this work that FFT was able to show the sidebands around the gearmesh frequencies. However, the background noise could cover make it difficult to observe the frequency contents of vibration signal accurate, potentially leading to inaccurate diagnostic. STFT did not perform well in indicating the location of the faulty gear although it was still able to distinguish between the normal and faulty gearbox from its timefrequency representation. However, STFT could be used as a fault indicator when there was no fault in the gear. Finally, CWT combined with the TSA could provide satisfactory results by not only be able to distinguish the fault pattern from faulty gearbox compared to a healthy gearbox but could also provide a reasonably accurately angular location of the faulty tooth at around $270^{\circ}$.

\section{Acknowledgment}

The authors gratefully acknowledge the support provided by Ningbo Science and Technology Bureau-International Cooperation Programme (Project: 2012D10029), China.

\section{References}

1. J. Bao, B. Tang, J. Zhao, Msrmnt 83, 57-71 (2016)

2. B. Liang, S. D. Iwnicki, Y. Zhao, Mech. Sys. Sig. Proc 39, 342-360 (2013)

3. G. He, K. Ding, W. Li. X. Jiao, Ren. Engy 87, 364375 (2016)

4. Y. Qin, B. Tang, Y. Mao, Sig. Proc 120, 480-494 (2016)

5. Y, Yang, X. J. Dong, Z. K. Peng, W. M. Zhang, G. Meng, J. Snd. Vib 335, 350-366 (2015)

6. G. Dalpiaz, A. Rivola, R. Rubini, Mech. Sys. Sig. Proc 14, 387-412 (2000)

7. G. He, K. Ding, H. Lin, Mech. Sys. Sig. Proc 66-67, 597-611 (2016)

8. N. H. Chandra, A. S. Sekhar, Mech. Sys. Sig. Proc 72-73, 105-133 (2016)

9. J. Chen, Z. Li, J. Pan, G. Chen, Y. Zi, J. Yuan, B. Chen, Z. He, Mech. Sys and Sig. Proc 70-71, 1-35 (2016)

10. W. He, Q. Miao, M. Azarian, M. Pecht, Mech. Sys. Sig. Proc 64-65, 149-161 (2015)

11. W. Liu, B. Tang, Exp. Sys. Appl 38, 7575-7581 (2011)

12. P. He, P. Li, H. Sun, Proc. Eng 15, 464-468 (2011)

13. L. Jedlinski, J. Jonak, Appl. Sft. Cmpt 30, 636-641 (2015) 